\title{
BMJ Open Clinical characteristics, management and 1-year outcomes of patients with acute coronary syndrome in Iran: the Iranian Project for Assessment of Coronary Events 2 (IPACE2)
}

Seyed Ebrahim Kassaian, ${ }^{1}$ Farzad Masoudkabir, ${ }^{1}$ Hashem Sezavar, ${ }^{2}$ Mohammad Mohammadi, ${ }^{3}$ Ali Pourmoghaddas, ${ }^{4}$ Javad Kojouri, ${ }^{5}$ Samad Ghaffari, ${ }^{6}$ Hamidreza Sanaati, ${ }^{7}$ Farshid Alaeddini, ${ }^{8}$ Bahin Pourmirza, ${ }^{9}$ Elham Mir, ${ }^{9}$ on-behalf of the IPACE2 registry investigators

To cite: Kassaian SE, Masoudkabir F, Sezavar H, et al. Clinical characteristics, management and 1-year outcomes of patients with acute coronary syndrome in Iran: the Iranian Project for Assessment of Coronary Events 2 (IPACE2). BMJ Open 2015;5:e007786. doi:10.1136/bmjopen-2015007786

- Prepublication history for this paper is available online. To view these files please visit the journal online (http://dx.doi.org/10.1136/ bmjopen-2015-007786).

Received 3 February 2015 Revised 6 October 2015 Accepted 29 October 2015

CrossMark

For numbered affiliations see end of article.

Correspondence to Dr Seyed Ebrahim Kassaian; ekassaian@yahoo.com

\section{ABSTRACT}

Objectives: To assess contemporary data on characteristics, management and 1-year postdischarge outcomes in Iranian patients hospitalised with acute coronary syndrome (ACS).

Setting: 11 tertiary care hospitals in 5 major cities in the Islamic Republic of Iran.

Participants: Patients aged $\geq 20$ and $\leq 80$ years discharged alive with confirmed diagnosis of ACS including ST-segment elevation myocardial infarction (STEMI), non-STEMI (NSTEMI) and high-risk unstable angina (HR-UA).

Primary and secondary outcome measures:

Patients were followed up regarding the use of medications and the end points of the study at 1 month and 1 year after discharge. The primary end point of the study was 1-year postdischarge major adverse cardiac and cerebrovascular events (MACCEs), defined as mortality (cardiac and non-cardiac), ACS and cerebrovascular attack (stroke and/or transient ischaemic attack). The secondary end points were hospital admission because of congestive heart failure, revascularisation by coronary artery bypass grafting surgery or percutaneous coronary intervention (PCl), and major and minor bleeds.

Results: A total of 1799 patients (25.7\% STEMI and $74.3 \%$ HR-UA/NSTEMI) discharged alive with confirmed diagnosis of ACS were included in the final analysis. During hospitalisation, the majority of the patients received aspirin $(98.6 \%)$, clopidogrel $(91.8 \%)$, anticoagulants $(93.4 \%)$, statins $(94.3 \%)$ and $\beta$-blockers $(89.3 \%)$. Reperfusion therapy was performed in $62.6 \%$ of patients with STEMI (46.3\% thrombolytic therapy and $17.3 \%$ primary $\mathrm{PCl}$ ). The mean door-to-balloon and door-to-needle times were 82.9 and $45.6 \mathrm{~min}$, respectively. In our study, $64.7 \%$ and $79.5 \%$ of the patients in HR-UA/NSTEMI and STEMI groups, respectively, underwent coronary angiography. During the 12 months after discharge, MACCEs occurred in $15.0 \%$ of all patients.

\section{Strengths and limitations of this study}

This is the first and only study on management and outcomes of patients with acute coronary syndrome (ACS) in Iran.

- In addition to assessment of in-hospital management, the patients were followed up for 1 year regarding the cardiovascular events and adherence to evidence-based treatment for ACS.

- We comprehensively compared the situation of Iran regarding management and outcome of ACS with that in developed countries and developing nations of the Middle East region.

- Although the study was multicentre and 11 hospitals participated, it was not a population-based registry and selection bias could have occurred.

Conclusions: Our study showed that the composition of Iranian patients with ACS regarding the type of ACS is similar to that in developed European countries and is unlike that in developing countries of the Middle East and Africa. We found that our patients with ACS are treated with high levels of adherence to guidelinerecommended in-hospital medications.

\section{INTRODUCTION}

Acute coronary syndrome (ACS) represents a major healthcare burden worldwide. The diagnosis and management of unstable angina (UA), non-ST-segment elevation myocardial infarction (NSTEMI) and STEMI have been rapidly evolving in recent years. ${ }^{1}$ However, ACS continues to be a significant health problem throughout the world, being responsible for a substantial number of 
deaths due to cardiovascular diseases (CVDs). The status of Middle Eastern nations in this context is especially worrying, as, according to prediction by the WHO, they will face the greatest increment in the absolute burden of CVD in the world. ${ }^{2}$

In recent years, well-regarded scientific societies in Europe and the USA have developed several guidelines to improve the outcomes of ACS through implementation of recommendations into clinical practice. ${ }^{3-6}$ Most of the real-world evidence about patients with ACS comes from several large registries ${ }^{7-12}$ with data on demography, treatments and outcomes of patients in middle-income and high-income countries, and little is known about patients with ACS in developing countries. Moreover, findings of the surveys and registries performed so far demonstrate that epidemiology and management of patients with ACS differ a lot between countries, and there is a wide gap between guidelines and current clinical practice. ${ }^{1} 7{ }^{14}$ Additionally, multinational registries often represent statistical averages for the participating centres rather than representing a real, existing geographical population. ${ }^{14}$ Hence, more representative local registries are needed in developing countries to increase awareness of CVD burden, its management and outcomes, in order to establish appropriate preventive and management strategies.

There are very limited data regarding the epidemiology, management and outcomes of ACS in Iranian patients. The Iranian Project for Assessment of Coronary Events 2 (IPACE2) was a prospective nationwide multicentre registry designed to gain insights into the epidemiology, clinical characteristics, management and 1-year postdischarge outcomes of Iranian patients hospitalised with ACS.

\section{MATERIALS AND METHODS}

\section{Study participants}

Between April 2011 and November 2012, we established a prospective multicentre registry that recruited patients aged $\geq 20$ and $\leq 80$ years with any type of ACS (STEMI, NSTEMI or high risk (HR)-UA) from 11 hospitals in five major cities in the Islamic Republic of Iran.

We obtained ethical approval before initiation of the study and all patients provided informed consent.

According to the protocol, all admitted patients with suspected ACS were screened to be eligible to enter the registry. However, we enrolled those patients with final diagnosis of ACS who were discharged alive from hospital and who gave informed consent for participation in the study. The final diagnosis was made by the attending cardiologist, based on clinical presentation, initial ECG pattern and markers of myocardial necrosis acquired at least $6 \mathrm{~h}$ after the symptom onset. The patients were then classified as having HR-UA, NSTEMI or STEMI. The definition of the final diagnosis was as follows:

- STEMI: presence of (1) ST-segment elevation consistent with myocardial infarction (MI) of $\geq 2 \mathrm{~mm}$ in adjacent chest leads and/or ST-segment elevation of $\geq 1 \mathrm{~mm}$ in two or more standard leads or new left bundle branch block (LBBB) and (2) positive cardiac necrosis markers.

- NSTEMI: (1) absence of ST-segment elevation consistent with MI of $\geq 2 \mathrm{~mm}$ in adjacent chest leads and ST-segment elevation of $\geq 1 \mathrm{~mm}$ in two or more standard leads and new LBBB and (2) positive cardiac necrosis markers.

- HR-UA: (1) absence of ST-segment elevation consistent with MI of $\geq 2 \mathrm{~mm}$ in adjacent chest leads and ST-segment elevation of $\geq 1 \mathrm{~mm}$ in two or more standard leads and new LBBB and (2) negative cardiac necrosis markers and (3) angina pectoris (or equivalent type of ischaemic discomfort) with any one of the three following features: (1) angina occurring at rest and prolonged, $>20 \mathrm{~min}$, (2) new-onset angina of at least Canadian Cardiovascular Society (CCS) class III severity or (3) recent acceleration of angina reflected by an increase in severity of at least one CCS class to at least CCS class III.

\section{Study protocol}

A detailed protocol was prepared with inclusion and exclusion criteria, methods and logistics, and definitions of all fields in the registry dataset. Also, the representative investigators from each collaborating hospital reviewed the workflow of the protocol in steering committee meetings before the registry was started. The patient with ACS registry was designed to collect data on demographic characteristics, medical history, cardiovascular risk factors, clinical presentation, time of symptom onset, early in-hospital management, reperfusion treatment, time of admission and start of thrombolysis or balloon, findings of diagnostic tests, hospital length of stay, discharge medications, and 1-month and 1-year follow-up for medications and outcomes.

Data for this registry were gathered at each centre by investigators instructed on the use of standardised electronic case report forms (e-CRF). All investigators had a username and password specific for them and were trained on how to extract and enter data to the electronic web-based registry. During the data collection, trained clinical audits supervised the compliance with study protocol and validity of the data. Moreover, the consistency and accuracy of data entry was overseen by a qualified independent assessor.

Patients were followed up regarding the use of medications through phone calls or direct visits at 1 month and 1 year after discharge as well as at the end points of the study. For the telephone follow-up interviews, at least five attempts were made to contact participants or their first-degree relatives. If telephone interviews were unsuccessful, the participants were contacted by mail using their home address. The primary endpoint of this study was 1-year postdischarge major adverse cardiac and cerebrovascular events (MACCEs), defined as mortality (cardiac and non-cardiac), ACS and cerebrovascular 
attack (CVA) (stroke and/or transient ischaemic attack, TIA). The secondary end points were hospital admission because of congestive heart failure, revascularisation by coronary artery bypass grafting (CABG) surgery or percutaneous coronary intervention (PCI), and major and minor bleeds. ACS was identified as HR-UA, NSTEMI or STEMI, using the aforementioned definitions, that resulted in readmission of the patient after discharge. CVA (stroke and/or TIA) was defined as an acute neurological deficit accompanied by brain imaging compatible with a recent ischaemic or haemorrhagic event. Major bleeding was defined as overt clinical bleeding: (1) that was associated with a drop in haemoglobin of more than $5 \mathrm{~g} / \mathrm{dL}$ or a haematocrit of $>15 \%$ (absolute); (2) that caused haemodynamic compromise or (3) that required a blood transfusion.

Adherence to guideline-recommended antiplatelet treatment was defined based on the recommendations of the "2011 American College of Cardiology (ACC) / American Heart Association (AHA) Focused Update of the Guidelines for the Management of Patients With Unstable Angina/Non-ST-Elevation Myocardial Infarction" 3 and "2009 Focused Updates of ACC/AHA Guidelines for the Management of Patients With ST-Elevation Myocardial Infarction," 4 for antiplatelet treatment on admission and after discharge in any subgroup of patients with ACS. Full adherence was defined as receiving antiplatelet treatment in compliance with the guideline at all time points (at discharge, 1 month after discharge and 1 year after discharge).

\section{Role of the funding source}

Sanofi-Aventis Groupe Iran Affiliate was the sponsor for this study. The sponsor had significant contribution in study design and preparation of the logistics. However, it had no role in data analysis, data interpretation or writing of the report. The Steering Committee, which was composed of investigators and an agent of the sponsor, was involved in the preparation and approval of the protocol, and supervised the conduct of study. The committee had full access to all the data in the study and was given full authority for presentation and publication of the results.

\section{Statistical analysis}

The Kolmogorov-Smirnov test was applied to examine normal distribution. The continuous variables are expressed as mean $\pm \mathrm{SD}$ and were compared between the STEMI/new LBBB and HR-UA/NSTEMI groups, using the Student t test. The categorical variables were compared using a $\chi^{2}$ test or the Fisher exact test, as appropriate, and they are presented as absolute frequencies with percentages. Multiple logistic regression analyses were used to determine independent predictors of MACCEs in patients with STEMI/new LBBB and also in patients with HR-UA/NSTEMI at 1 year after discharge. Variables were entered into the logistic regression model based on their statistical significance in univariable analyses (entering the criterion $\mathrm{p} \leq 0.1$ ) as well as on their clinical significance (based on the investigators' discretion). The final included variables in the model were heart rate on admission, positive histories of diabetes mellitus (DM), and/or peripheral arterial disease, PCI or CABG during the admission or 1-year postdischarge, congestive heart failure during admission or 1-year postdischarge, left ventricular ejection fraction (LVEF) $<40 \%$, left main disease or multivessel coronary artery disease in coronary angiography and full adherence to guideline-recommended antiplatelet therapy throughout the first year after ACS. Moreover, we also included sex, typical chest pain at presentation, history of CVA, reperfusion therapy and anterior STEMI in the STEMI/new LBBB group. For all analyses, the statistical package SPSS V.16.0 for Windows (SPSS Inc, Chicago, Illinois, USA) was used. All $p$ values were two-tailed with significance defined as $\mathrm{p} \leq 0.05$.

\section{RESULTS}

\section{Study population}

Between April 2011 and November 2012, 1997 patients with suspected ACS were recruited from 20 teaching hospitals in five major cities of Iran. Of the 1997 patients recruited, 1799 patients were discharged alive with confirmed diagnosis of ACS: 855 from Tehran (47.5\%); 377 from Mashhad (21.0\%); 167 from Tabriz (9.3\%); 206 from Isfahan (11.5\%) and 194 from Shiraz (10.8\%). One year follow-up was successfully completed in 1640 patients, for an overall follow-up rate of $91.2 \%$.

Discharge diagnoses were STEMI/new LBBB in 463 patients $(25.7 \%)$ and HR-UA/NSTEMI in 1336 patients $(74.3 \%)$. Of the patients with UA/NSTEMI, 377 (20.9\%) had NSTEMI and 959 (53.3\%) had HR-UA. Table 1 shows the baseline demographic and clinical characteristics of these groups. For the entire patient group, the mean $( \pm \mathrm{SD})$ age was $60.1( \pm 11.2)$ years and $65.4 \%$ were male. Patients with HR-UA/NSTEMI tended to have more concomitant diseases including hypertension, hyperlipidaemia, DM and histories of UA, MI, PCI, CABG and peripheral arterial disease than did patients with STEMI/new LBBB. However, patients with STEMI/ new LBBB were more likely to be men, younger and current smokers, and to present with typical ischaemic chest pain.

\section{In-hospital medications and interventions}

Table 2 shows the prescription of guideline-recommended medications in the first $24 \mathrm{~h}$ of admission in the entire patient group. Aspirin and clopidogrel were given to $98.6 \%$ and $91.8 \%$, respectively, and $91.2 \%$ of the patients received dual antiplatelet therapy (DAPT). Patients with STEMI/new LBBB were more likely to receive clopidogrel as well as DAPT than were patients with HR-UA/NSTEMI. Overall, $94.3 \%$ of the patients were treated with statins and $89.3 \%$ received $\beta$-blockers. ACE inhibitors or angiotensin receptor blockers were 
Table 1 Baseline demographic and clinical characteristics of the study patients

\begin{tabular}{|c|c|c|c|c|}
\hline Characteristics & Total $(n=1799)$ & STEMI/new LBBB $(n=463)$ & UA/NSTEMI $(n=1336)$ & p Value \\
\hline \multicolumn{5}{|l|}{ Demographics } \\
\hline Age, year & $60.1 \pm 11.2$ & $58.8 \pm 11.3$ & $60.5 \pm 11.1$ & 0.004 \\
\hline Male sex, n (\%) & $1177(65.4)$ & $371(80.1)$ & $806(60.3)$ & $<0.0001$ \\
\hline \multicolumn{5}{|l|}{ Presenting characteristics } \\
\hline Ischaemic-type chest pain, n (\%) & $1638(91.1)$ & $445(96.1)$ & 1193 (89.3) & $<0.0001$ \\
\hline Cardiac arrest/ASCD, $\mathrm{n}(\%)$ & $2(0.1)$ & $2(0.4)$ & $0(0.0)$ & 0.066 \\
\hline Heart rate, bpm & $78.6 \pm 17.3$ & $78.7 \pm 16.6$ & $78.5 \pm 17.6$ & 0.829 \\
\hline $\mathrm{SBP}, \mathrm{mm} \mathrm{Hg}$ & $135.4 \pm 25.6$ & $133.9 \pm 26.7$ & $135.9 \pm 25.2$ & 0.140 \\
\hline $\mathrm{DBP}, \mathrm{mm} \mathrm{Hg}$ & $82.2 \pm 15.0$ & $82.6 \pm 16.2$ & $82.1 \pm 14.6$ & 0.585 \\
\hline \multicolumn{5}{|l|}{ Risk factors, n (\%) } \\
\hline Hypertension & 898 (49.9) & $183(39.5)$ & $715(53.6)$ & $<0.0001$ \\
\hline Hyperlipidaemia & $826(45.9)$ & 159 (34.9) & $667(50.5)$ & $<0.0001$ \\
\hline Diabetes mellitus & $559(31.1)$ & $120(25.9)$ & 439 (32.9) & $<0.0001$ \\
\hline Family history of CAD & $510(28.4)$ & $119(27.4)$ & 391 (31.0) & 0.150 \\
\hline \multicolumn{5}{|l|}{ Smoking } \\
\hline Current & $530(29.5)$ & $199(43.0)$ & $331(24.8)$ & $<0.0001$ \\
\hline Past & $184(10.2)$ & $41(8.9)$ & $143(10.7)$ & \\
\hline \multicolumn{5}{|l|}{ Medical history, n (\%) } \\
\hline UA & 797 (44.3) & $119(26.0)$ & 678 (51.4) & $<0.0001$ \\
\hline MI & 330 (18.3) & $60(13.1)$ & $270(20.6)$ & $<0.0001$ \\
\hline $\mathrm{PCl}$ & 196 (10.9) & $31(6.7)$ & $165(12.4)$ & 0.001 \\
\hline CABG & $158(8.8)$ & $14(3.0)$ & $144(10.8)$ & $<0.0001$ \\
\hline CVA & $93(5.2)$ & $20(4.3)$ & $73(5.5)$ & 0.324 \\
\hline PAD & $30(1.7)$ & $3(0.7)$ & $27(2.3)$ & 0.034 \\
\hline
\end{tabular}

All plus-minus values are mean $\pm \mathrm{SD}$.

ASCD, aborted sudden cardiac death; CABG, coronary artery bypass grafting surgery; CAD, coronary artery disease; CVA, cerebrovascular attack; DBP, diastolic blood pressure; LBBB, left bundle branch block; MI, myocardial infarction; NSTEMI, non-ST-segment elevation myocardial infarction; PAD, peripheral arterial disease; PCI, percutaneous coronary intervention; SBP, systolic blood pressure; STEMI,

ST-segment elevation myocardial infarction; UA, unstable angina.

administered in $81.9 \%$ of all patients, and $93.4 \%$ received anticoagulation therapy with almost similar proportions of intravenous unfractionated heparin and subcutaneous low-molecular-weight heparin.

Among patients with STEMI/new LBBB, 290 $(62.6 \%)$ underwent reperfusion therapy; $46.3 \%$ of the patients with STEMI/new LBBB received thrombolytic therapy, which was mostly streptokinase (table 3), and primary PCI was carried out in $17.3 \%$ of the patients with STEMI/new LBBB. The mean door-to-balloon and door-to-needle times were 82.9 and $45.6 \mathrm{~min}$, respectively. Iranian patients with ACS had a mean 265.6 min delay from symptom onset to presenting to a hospital.

Table 2 In-hospital medication administered for the study patients

\begin{tabular}{|c|c|c|c|c|}
\hline Characteristics & Total $(n=1799)$ & STEMI/new LBBB $(n=463)$ & UA/NSTEMI ( $n=1336)$ & p Value \\
\hline \multicolumn{5}{|l|}{ Medications, n (\%) } \\
\hline Aspirin & $1773(98.6)$ & $460(99.4)$ & 1313 (98.3) & 0.095 \\
\hline Clopidogrel & $1652(91.8)$ & $454(98.1)$ & 1198 (89.7) & $<0.0001$ \\
\hline Other antiplatelets & $15(0.8)$ & $6(1.3)$ & $9(0.7)$ & 0.235 \\
\hline Dual antiplatelet therapy & 1640 (91.2) & $452(97.6)$ & $1188(88.9)$ & $<0.0001$ \\
\hline UFH & 864 (48.0) & $230(49.7)$ & 634 (47.5) & 0.410 \\
\hline LMWH & 817 (45.4) & $197(42.5)$ & $620(46.4)$ & 0.151 \\
\hline Statin & 1697 (94.3) & 441 (95.2) & $1256(94.0)$ & 0.322 \\
\hline$\beta$-blocker & 1606 (89.3) & 421 (90.9) & $1185(88.7)$ & 0.181 \\
\hline ACEI/ARB & 1473 (81.9) & $403(87.0)$ & $1070(80.1)$ & 0.001 \\
\hline Nitrates & 1653 (91.9) & $417(90.1)$ & $1236(92.5)$ & 0.096 \\
\hline Oral antiglycaemic agents & $228(12.7)$ & $36(7.8)$ & $192(14.4)$ & $<0.0001$ \\
\hline PPls & $746(41.5)$ & $206(44.5)$ & $540(40.4)$ & 0.125 \\
\hline
\end{tabular}

ACEI, ACE inhibitor; ARB, angiotensin receptor blocker; LBBB, left bundle branch block; LMWH, low-molecular-weight heparin; NSTEMI, non-ST-segment elevation myocardial infarction; PPIs, proton-pump inhibitors; STEMI, ST-segment elevation myocardial infarction; UA, unstable angina; UFH, unfractionated heparin. 
Table 3 In-hospital reperfusion and revascularisation strategies in the study patients

\begin{tabular}{|c|c|c|c|c|}
\hline Characteristics & Total $(n=1799)$ & STEMI/new LBBB $(n=463)$ & UA/NSTEMI $(n=1336)$ & p Value \\
\hline \multicolumn{5}{|l|}{ Reperfusion strategies, n (\%) } \\
\hline No reperfusion & - & $153(33.6)$ & - & - \\
\hline Primary CABG & - & $13(2.9)$ & - & - \\
\hline Thrombolytic therapy & - & $211(46.3)$ & - & - \\
\hline Symptom onset to thrombolytic & - & $269.0 \pm 477.3$ & - & - \\
\hline Door-to-needle time & - & $45.6 \pm 41.1$ & - & - \\
\hline Primary $\mathrm{PCl}$ & - & $79(17.3)$ & - & - \\
\hline Symptom onset to $\mathrm{PCl}$ & - & $256.8 \pm 186.6$ & - & - \\
\hline Door-to-balloon time & - & $82.8 \pm 112.5$ & - & - \\
\hline \multicolumn{5}{|l|}{ Revascularisation strategies, $\mathrm{n}(\%)$} \\
\hline Coronary angiography & $1232(68.5)$ & $368(79.5)$ & $864(64.7)$ & $<0.0001$ \\
\hline $\mathrm{PCl}$ (excluding primary $\mathrm{PCl}$ ) & $360(20.0)$ & $133(28.7)$ & $227(17.0)$ & $<0.0001$ \\
\hline DES & $258(71.9)$ & $94(70.1)$ & $164(72.9)$ & 0.764 \\
\hline BMS & $81(22.6)$ & $33(24.6)$ & $48(21.3)$ & \\
\hline Both & $20(5.5)$ & $7(5.2)$ & $13(5.8)$ & \\
\hline CABG surgery & $180(10.0)$ & $34(7.3)$ & $146(10.9)$ & 0.049 \\
\hline
\end{tabular}

All plus-minus values are mean \pm SD.

BMS, bare metal stent; CABG, coronary artery bypass grafting surgery; DES, drug-eluting stent; LBBB, left bundle branch block; NSTEMI, non-ST-segment elevation myocardial infarction; PCI, percutaneous coronary intervention; STEMI, ST-segment elevation myocardial infarction; UA, unstable angina.

In the HR-UA/NSTEMI group, invasive strategy (coronary angiography) was applied in about two-thirds of the patients, while $79.5 \%$ of the patients in STEMI/new LBBB group underwent coronary angiography. Elective PCI during hospital stay was performed in $20.0 \%$ of all patients. Among the patients who underwent PCI, $71.9 \%$ received drug-eluting stents, $22.6 \%$ received baremetal stents and 5.5\% received both types of stents. During the hospital stay, $10.0 \%$ of the patients underwent CABG surgery.

\section{Postdischarge adherence to antiplatelet therapy}

Table 4 shows the compliance with guideline-recommended antiplatelet treatment for patients with ACS at discharge, and 1 month and 1 year after discharge. At discharge, $77.5 \%$ of the patients with STEMI/new LBBB received guideline-compliant antiplatelets, but this amount gradually decreased and only about half of the patients with STEMI/new LBBB had full adherence to guideline-recommended antiplatelet treatment at 1 year. In the HR-UA/NSTEMI group, $67.0 \%$ were discharged with guideline-recommended antiplatelet regimens, but only $28.3 \%$ of the patients were using antiplatelets in compliance with the guideline for the entire 12 months after discharge. Moreover, at discharge and 1 and 12 months after discharge, the patients who had undergone PCI or CABG during index hospitalisation were more likely than patients scheduled for conservative

Table 4 Adherence to postdischarge guideline-recommended antiplatelet therapy

\begin{tabular}{|c|c|c|c|c|c|c|c|c|}
\hline & \multicolumn{2}{|c|}{ At discharge } & \multicolumn{2}{|c|}{$\begin{array}{l}1 \text { Month after } \\
\text { discharge }\end{array}$} & \multicolumn{2}{|c|}{$\begin{array}{l}1 \text { Year after } \\
\text { discharge }\end{array}$} & \multicolumn{2}{|c|}{ Full adherence } \\
\hline & Yes & No & Yes & No & Yes & No & Yes & No \\
\hline STEMI/new LBBB & $359(77.5)$ & $104(22.5)$ & $322(72.4)$ & $123(27.6)$ & $207(51.8)$ & $193(48.3)$ & $197(49.7)$ & $199(50.3)$ \\
\hline Revascularisation* & $210(85.0)$ & $37(15.0)$ & 193 (80.4) & 47 (19.6) & $141(64.1)$ & 79 (35.9) & $139(63.5)$ & $80(36.5)$ \\
\hline $\begin{array}{l}\text { Conservative } \\
\text { management }\end{array}$ & $149(69.0)$ & $67(31.0)$ & 129 (62.9) & $76(37.1)$ & $66(36.7)$ & 114 (63.3) & $58(32.8)$ & $119(67.2)$ \\
\hline UA/NSTEMI & $895(67.0)$ & $441(33.0)$ & $753(58.6)$ & $533(41.4)$ & $424(37.9)$ & $695(62.1)$ & $313(28.3)$ & $792(71.7)$ \\
\hline Revascularisation* & $300(77.7)$ & $86(22.3)$ & 288 (76.6) & $88(23.4)$ & $156(46.0)$ & $183(54.0)$ & 105 (31.3) & $230(68.7)$ \\
\hline $\begin{array}{l}\text { Conservative } \\
\text { management }\end{array}$ & $595(62.6)$ & $355(37.4)$ & $465(51.1)$ & $445(48.9)$ & $268(34.4)$ & $512(65.6)$ & $208(27.0)$ & $562(73.0)$ \\
\hline Total & 1254 (69.7) & $545(30.3)$ & $1075(62.1)$ & $656(37.9)$ & $631(41.5)$ & $888(58.5)$ & $510(34.0)$ & $991(66.0)$ \\
\hline Revascularisation* & 510 (80.6) & $123(80.6)$ & 481 (78.1) & $135(21.9)$ & $297(53.1)$ & 262 (46.9) & $244(44.0)$ & $310(56.0)$ \\
\hline $\begin{array}{l}\text { Conservative } \\
\text { management }\end{array}$ & $744(63.8)$ & $422(36.2)$ & 594 (53.3) & $521(46.7)$ & 334 (34.8) & 626 (65.2) & $266(28.1)$ & $681(71.9)$ \\
\hline
\end{tabular}


management to receive antiplateletes according to the ACC/AHA recommendations for antiplatelet treatment in patients with ACS.

\section{Postdischarge outcomes}

Table 5 demonstrates the 1-year postdischarge outcomes of the study patients. During the entire study period, 70 patients died (22 in the STEMI/new LBBB group and 48 in the HR-UA/NSTEMI group). Although 1-year total postdischarge mortality did not show significant difference between the two groups, the cardiovascular mortality was higher in patients with STEMI/new LBBB than in patients with HR-UA/NSTEMI, with statistically borderline significance $(5.2 \%$ vs $3.9 \%$, respectively, $\mathrm{p}=0.061)$. Moreover, patients with STEMI/new LBBB were more likely to undergo PCI $(15.9 \%$ vs $7.7 \%)$ or CABG $(9.0 \%$ vs $6.5 \%)$ after discharge. During the 12-months follow-up, MACCEs occurred in $15.0 \%$ of all patients and the rate of the MACCE was similar in the STEMI/new LBBB and HR-UA/NSTEMI groups. Of the entire patient group, $1.4 \%$ experienced at least one episode of bleeding during 1-year follow-up, of which $30.4 \%$ were major bleeds. Patients with STEMI/new LBBB and HR-UA/NSTEMI were found to have similar rates of bleeding, and of similar severity, during the 12 months after discharge.

\section{Predictors of a 1-year postdischarge MACCE}

Table 6 shows the independent predictors of MACCEs during 1-year follow-up in the STEMI/new LBBB and HR-UA/NSTEMI groups separately. In the patients with STEMI/new LBBB, LVEF $\leq 40 \%$ was associated with increased risk of a MACCE at 1-year postdischarge (OR $1.69 ; 95 \%$ CI, 1.03 to $2.75, \mathrm{p}=0.036$ ). Typical ischaemic chest pain at presentation was independently associated with lower risk of a MACCE at 12 months after discharge in patients with STEMI (OR 0.24; 95\% CI, 0.09 to 0.62 , $\mathrm{p}=0.003)$. CABG during the index hospitalisation or later during the first year after discharge was associated with lower risk of a MACCE at 1 year but the statistical significance was borderline (OR 0.27, p=0.087). Among the patients with HR-UA/NSTEMI, (a positive history of) DM, a high heart rate at presentation and history of PCI were associated with significantly increased risk of a MACCE at 12 months after discharge; revascularisation during index hospitalisation was, rather, associated with a lower MACCE risk (OR 0.356, 95\% CI, 0.233 to 0.543 , $\mathrm{p}<0.0001)$.

\section{DISCUSSION}

The IPACE2 study is the first to evaluate the clinical characteristics, and contemporary diagnostic and therapeutic strategies applied to patients with ACS in Iran. In addition, this survey sheds light on the mid-term prognosis and its predictors in a wide spectrum of 'real world' Iranian patients with ACS.

In our study, almost one-fourth (25.7\%) of patients with ACS had STEMI, which is similar to proportions reported from developed countries, ${ }^{14-16}$ and it is significantly lower than the values reported from Gulf countries $(45.6 \%)$, India $(60 \%)$, and developing countries in Latin America and Africa ${ }^{17-20}$ (table 7). This outcome is likely to be the result of overall younger age (56-57 vs 60.1 years, respectively) and also higher male/female ratio of the patients with ACS in these countries than in ours (3.73 vs 1.89 , respectively). Moreover, several factors in addition to the younger age of the population in Arabian countries-including the significantly higher prevalence of DM $(39.5 \%$ vs $31.1 \%)$ and current or past smoking $(52.9 \%$ vs $39.7 \%)$-in their studied population, not as considerable in ours, may be accountable for the observed difference. However, with a mean age of 60.1 years, participants in

\begin{tabular}{|c|c|c|c|c|}
\hline Characteristics & Total $(n=1640)$ & STEMI/new LBBB $(n=421)$ & UA/NSTEMI $(n=1219)$ & p Value \\
\hline Mortality & $70(4.3)$ & $22(5.2)$ & $48(3.9)$ & 0.267 \\
\hline Cardiac & $50(71.4)$ & $19(86.4)$ & $31(64.6)$ & 0.061 \\
\hline Non-cardiac & $20(28.6)$ & 3 (13.6) & $17(35.4)$ & \\
\hline Acute coronary syndrome & $156(9.5)$ & $36(8.5)$ & $120(9.8)$ & 0.427 \\
\hline Congestive heart failure & 54 (3.3) & $12(2.8)$ & $42(3.4)$ & 0.551 \\
\hline CABG & $117(7.1)$ & $38(9.0)$ & $79(6.5)$ & 0.081 \\
\hline $\mathrm{PCl}$ & $161(9.8)$ & $67(15.9)$ & $94(7.7)$ & $<0.0001$ \\
\hline Stroke/TIA & $20(1.2)$ & $5(1.2)$ & $15(1.2)$ & 0.940 \\
\hline Bleeding & $23(1.4)$ & $6(1.4)$ & $17(1.4)$ & 0.969 \\
\hline Severe & $7(30.4)$ & 2 (33.3) & $5(29.4)$ & \\
\hline Moderate & $4(17.4)$ & $1(16.7)$ & $3(17.6)$ & \\
\hline Mild & $12(52.2)$ & $3(50.0)$ & 9 (52.9) & \\
\hline MACCE $^{*}$ & $246(15.0)$ & 63 (14.9) & $183(15.0)$ & 0.996 \\
\hline
\end{tabular}


Table 6 Independent predictors of the major adverse cardiovascular events during 1-year postdischarge in patients with STEMI/new LBBB and HR-UA/NSTEMI

\begin{tabular}{|c|c|c|c|c|}
\hline \multirow[b]{2}{*}{ Variable } & \multicolumn{2}{|l|}{ STEMI/new LBBB } & \multicolumn{2}{|l|}{ UA/NSTEMI } \\
\hline & OR (95\% Cl) & p Value & OR (95\% Cl) & p Value \\
\hline Ischaemic-type chest pain & 0.24 (0.09 to 0.62$)$ & 0.003 & - & - \\
\hline Heart rate $(\mathrm{bpm})$ & - & - & $1.01(1.000$ to 1.016$)$ & 0.048 \\
\hline Diabetes mellitus & - & - & $2.23(1.64$ to 3.03$)$ & $<0.0001$ \\
\hline $\mathrm{Hx}$ of $\mathrm{PCl}$ before admission & - & - & 1.61 (1.06 to 2.44$)$ & 0.025 \\
\hline Revascularisation* & - & - & $0.356(0.233$ to 0.543$)$ & $<0.0001$ \\
\hline LVEF $<40 \%$ & 1.69 (1.03 to 2.75$)$ & 0.036 & - & - \\
\hline
\end{tabular}

our study were significantly younger than those in the developed countries. ${ }^{114} 16172122$

The latest ACC/AHA guidelines for management of patients with STEMI recommends $\leq 30 \mathrm{~min}$ for door-to-needle time, $\leq 90 \mathrm{~min}$ for door-to-balloon time and $\leq 120 \mathrm{~min}$ for total ischaemic time, as the goal of reperfusion timing. ${ }^{4}$ Although our patients with STEMI were reperfused within acceptable lengths of time with respect to median door-to-needle time (mean: $45.6 \mathrm{~min} /$ median: $30 \mathrm{~min}$ ) and door-to-balloon time (mean: $82.8 \mathrm{~min}$, median: $53 \mathrm{~min}$ ), it took an average of about 265 min (median: $160 \mathrm{~min}$ ) for our patients with STEMI to reach the emergency department, which is higher than median times reported from developed countries. ${ }^{1} 1621$ The causes responsible for long delays before hospital arrival of patients with STEMI in the Iranian population should be elucidated in future studies.

Similar to recent trends reported by other ACS registries, ${ }^{1} 810161721$ 23-26 our study showed high compliance with guideline-recommended medications in the first $24 \mathrm{~h}$ of admission in Iranian patients with ACS, which demonstrates the good knowledge, attitude and practice of Iranian physicians with respect to the guideline-recommended in-hospital management of patients with ACS.

Despite the established beneficial effect of DAPT on outcomes of ACS and current ACC/AHA recommendations regarding antiplatelet treatment in patients with $\mathrm{ACS},{ }^{3}{ }^{4}$ global experiences have shown underutilisation of dual antiplatelet agents in patients with ACS, especially in those who were diagnosed as having acute MI

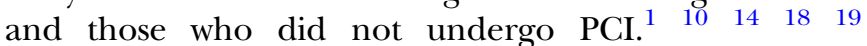
24252728 In our study, $91.8 \%$ of all patients, including $98.1 \%$ of the patients with STEMI/new LBBB and $89.7 \%$ of the patients with HR-UA/NSTEMI, received clopidogrel during the hospitalisation for ACS, which is significantly higher than previously reported values from other registries. ${ }^{1} 10 \quad 14 \quad 18 \quad 1924 \quad 25 \quad 27 \quad 28$ However, only $69.7 \%$ of our patients with ACS, including $77.5 \%$ of the patients with STEMI/new LBBB and $67.0 \%$ of the patients with HR-UA/NSTEMI, were prescribed DAPT at discharge, and these values progressively declined over time. In the IPACE2 study, only half of the patients in the STEMI/new LBBB group and one-third of the patients in the HR-UA/NSTEMI group who were discharged with DAPT, completed the treatment for 1 year. Given the weight of evidence supporting DAPT use throughout the wide spectrum of ACS, there is a substantial opportunity to reinforce the appropriate use of these medications in the management of the spectrum of ACS to improve clinical outcome.

In this study, we observed that patients with STEMI/ new LBBB and HR-UA/NSTEMI had similar incidence of all-cause mortality and MACCE during the year after discharge. However, cardiovascular mortality was significantly higher in patients with STEMI/new LBBB than in patients with HR-UA/NSTEMI and, conversely, noncardiac mortality was higher among patients with HR-UA/NSTEMI than among the STEMI/new LBBB group. This finding is in agreement with Polonski et al, ${ }^{14}$ who observed an adjusted worse long-term prognosis in patients with STEMI than that in patients with NSTEMI. The reasons for higher mid-term non-cardiac mortality in patients with HR-UA/NSTEMI might be: (1) older age of patients with HR-UA/NSTEMI than those in the STEMI/new LBBB group and (2) higher prevalence of major comorbidities in the HR-UA/NSTEMI group, such as DM, hypertension and hyperlipidaemia, which are associated with worse prognosis and mortality.

In this study, we observed that presentation of STEMI with a typical ischaemic chest pain was associated with a decreased risk of a MACCE at 1-year postdischarge. This finding supports and expands the findings by Canto et $a l^{29}$ which revealed that patients with MI without typical chest pain were less quickly diagnosed and treated, and had higher adjusted odds of hospital mortality, regardless of whether they had ST-segment elevation. The authors observed that patients without typical chest pain/discomfort were less likely to receive medications with established survival benefits and/or undergo timely reperfusion. ${ }^{29}$ In this IPACE2 study, we observed that DM significantly increased the risk of 1-year postdischarge MACCE in patients with HR-UA/NSTEMI. 
Table 7 Comparison of baseline characteristics, management and outcomes of acute coronary syndrome patients in developed and developing countries

\begin{tabular}{|c|c|c|c|c|c|c|c|c|c|c|c|}
\hline & NRMI & $\begin{array}{l}\text { Expanded } \\
\text { GRACE }\end{array}$ & EHS 2 & PL-ACS & $\begin{array}{l}\text { Portuguese } \\
\text { Registry of } \\
\text { ACS }\end{array}$ & PACIFIC & CREATE & SPACE & ACCESS & $\begin{array}{l}\text { GULF- } \\
\text { RACE2 }\end{array}$ & IPACE2 \\
\hline Sampling Period & 1990-2006 & 2001-2007 & 2004 & 2003-2006 & 2002-2008 & 2008-2009 & 2002-2005 & 2005-2007 & 2007-2008 & 2008-2009 & 2011-2012 \\
\hline Source of data & USA & Multinational & $\begin{array}{l}\text { Europe and } \\
\text { mediterranean } \\
\text { basin }\end{array}$ & Poland & Portugal & Japan & India & $\begin{array}{l}\text { Saudi } \\
\text { Arabia }\end{array}$ & $\begin{array}{l}\text { Developing } \\
\text { countries }\end{array}$ & $\begin{array}{l}\text { Gulf } \\
\text { countries }\end{array}$ & Iran \\
\hline Patients, $\mathrm{n}$ & 2515106 & 31982 & 6385 & 100193 & 22482 & 3597 & 31982 & 5055 & 12068 & 7930 & 1799 \\
\hline Mean age, years & 65 & 65 & 64 & 65 & 66 & 67 & 57 & 58 & 59 & 56 & 60 \\
\hline Male, \% & 58 & NA & 70 & 60 & 70 & 77 & 76 & 77 & 73 & 79 & 65 \\
\hline Current smoking, \% & 24 & NA & 37 & 27 & 24 & NS & 28 & 33 & 40 & 36 & 29 \\
\hline $\mathrm{DM}, \%$ & 29 & 26 & 24 & 23 & 27 & 35 & 30 & 58 & 24 & 39 & 31 \\
\hline STEMI, \% & 47 & 30 & 47 & 31 & 45 & 59 & 61 & 41.5 & 46 & 46 & 26 \\
\hline UA/NSTEMI, \% & 53 & 70 & 53 & 69 & 55 & 41 & 39 & 58.5 & 54 & 54 & 74 \\
\hline $\begin{array}{l}\text { Symptom onset to } \\
\text { hospital arrival time, } \\
\text { (min)* }\end{array}$ & 96 & 133 & 145 & 260 & 177 & NA & 300 & 150 & 240 & 176 & 160 \\
\hline $\begin{array}{l}\text { Door-to-needle } \\
\text { time },(\min )^{\star}\end{array}$ & 29 & 32 & 37 & 25 & 60 & NA & 50 & 52 & NA & 39 & 30 \\
\hline $\begin{array}{l}\text { Door-to-balloon } \\
\text { time, }(\min )^{*}\end{array}$ & 79 & 110 & 70 & 50 & 96 & NA & NA & NA & NA & 87 & 53 \\
\hline $\begin{array}{l}\text { Thrombolytic } \\
\text { therapy, \% }\end{array}$ & 28 & 33 & 41 & 9.3 & 44 & NA & 58.5 & 69.1 & 39 & 66 & 46 \\
\hline Primary $\mathrm{PCI}, \%$ & 43 & 16 & 58 & 54 & 19 & 63 & 8 & 17.5 & 22 & 22.3 & 17 \\
\hline $\begin{array}{l}\text { Coronary } \\
\text { angiography†, \% }\end{array}$ & 78 & 70 & 66 & 39 & 61 & 96 & 23 & 67 & 58 & 32 & 68 \\
\hline 30-day mortality, \% & NA & NA & $6.4 / 3.4$ & NA & NA & NA & 8.6/3.8 & NA & $5 / 2.4$ & $9.9 / 5$ & $2.5 / 1.0$ \\
\hline 1-year mortality, \% & NA & NA & 7.5 & NA & NA & $3 / 2.2$ & NA & NA & 8.4/6.3 & $11.5 / 7.7$ & $5.2 / 3.9$ \\
\hline
\end{tabular}

*Times in patients with STEMI.

tDuring index hospitalisation.

CREATE, treatment and outcomes of acute coronary syndromes in India; DM, diabetes mellitus; EHS2, European heart survey II; GRACE, global registry of acute coronary events;

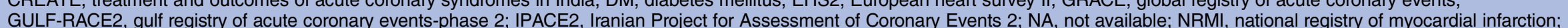

NS, not significant; PACIFIC, prevention of atherothrombotic incidents following ischemic coronary attack; PCl, percutaneous coronary intervention; PL-ACS, Polish Registry of Acute Coronary

Syndromes; SPACE, Saudi project for assessment of coronary events; STEMI, ST-segment elevation myocardial infarction; UA/NSTEMI, unstable angina/non-ST-segment elevation myocardial

infarction. 
Our finding supports and expands the study by Chong et $a l,{ }^{30}$ which observed that DM increases the risk of a MACCE at 1 and 6 months after discharge in patients with UA/NSTEMI. Park $e t a l^{31}$ also observed that DM is a predictor of early and late cardiac death in patients with NSTEMI (and not STEMI). This suggests that, among the traditional risk factors, presence of diabetes may be the most predictive factor for adverse clinical events after discharge in patients with HR-UA/NSTEMI.

The merits of our study are that it is the first and only study on management and outcomes of patients with ACS in Iran. However, our study had several limitations. First, although the study was multicentred, with 20 hospitals participating, it was not a population-based registry and selection bias could have occurred. Second, there is an inherent selection bias because of the observational nature of the study design and the possibility of important unmeasured covariables having been missed. Finally, although we compared our data with other international ACS registries, caution has to be taken about making absolute inferences, mainly because of the patient age and timing differences between these studies and ours.

In conclusion, the IPACE2 study showed that composition of Iranian patients with ACS regarding the type of ACS is similar to that in developed European countries and is unlike that in developing countries of the Middle East and Africa. We found that our patients with ACS are treated with high levels of adherence to guideline-recommended in-hospital medications, but there was a substantial underuse of DAPT at discharge, and it also progressively declined over time after discharge. Moreover, Iranian patients with STEMI delayed a long time before presenting to the hospital, but in-hospital reperfusions were quite timely.

\section{Author affiliations}

${ }^{1}$ Department of Cardiology, Tehran Heart Center, Tehran University of Medical Sciences, Tehran, Iran

${ }^{2}$ Department of Cardiology, Rasul-e-Akram Hospital, Iran University of Medical Sciences, Tehran, Iran

${ }^{3}$ Department of Cardiology, Javad-Al-Aemmeh Heart Hospital \& Research Center, Mashhad, Iran

${ }^{4}$ Department of Cardiology, Khorshid Hospital, Isfahan University of Medical Sciences, Isfahan, Iran

${ }^{5}$ Cardiovascular Research Center, Shiraz University of Medical Sciences, Shiraz, Iran

${ }^{6}$ Cardiovascular Research Center, Tabriz University of Medical Sciences, Tabriz, Iran

${ }^{7}$ Department of Cardiology, Rajaie Cardiovascular Medical and Research Center, Iran University of Medical Sciences, Tehran, Iran

${ }^{8}$ Department of Research, Tehran Heart Center, Tehran University of Medical Sciences, Tehran, Iran

${ }^{9}$ Sanofi Iran Medical Department, Tehran, Iran

Acknowledgements The authors would like to acknowledge Dr Abdollah Amirfarhangi, (Rasul-e-Akram Hospital, Iran University of Medical Sciences, Tehran), Dr Shekoufeh Hajsadeghi (Rasul-e-Akram Hospital, Iran University of Medical Sciences, Tehran), and Dr Majid Haghjoo and Dr Farshad Shakerian (Rajaie Cardiovascular Medical and Research Center, Iran University of Medical Sciences, Tehran), for their substantial contribution to this study as steering committee members. They also thank Dr Ayda Biria for her efforts in managing data collection and clearance.
Contributors SEK contributed to study design, management of data collection, data interpretation and editing the article. FM was the scientific consultant of the project and drafted the article. HS, MM, JK, SG and HS were members of the steering committee, and contributed to study design, data collection and critical review of the article. They were also the local coordinators of data collection in the participating centres. FA served as epidemiology and biostatistics consultant and performed the data analysis. $\mathrm{BP}$ and EM were the executive managers of the project and were involved in study design, coordination of the steering committee and monitoring of the project.

Funding This study was sponsored by Sanofi-Aventis Groupe Iran Affiliate.

Competing interests None declared.

Provenance and peer review Not commissioned; externally peer reviewed.

Data sharing statement No additional data are available.

Open Access This is an Open Access article distributed in accordance with the Creative Commons Attribution Non Commercial (CC BY-NC 4.0) license, which permits others to distribute, remix, adapt, build upon this work noncommercially, and license their derivative works on different terms, provided the original work is properly cited and the use is non-commercial. See: http:// creativecommons.org/licenses/by-nc/4.0/

\section{REFERENCES}

1. Mandelzweig L, Battler A, Boyko V, et al. The second Euro Heart Survey on acute coronary syndromes: characteristics, treatment, and outcome of patients with ACS in Europe and the Mediterranean Basin in 2004. Eur Heart J 2006;27:2285-93.

2. Wild S, Roglic G, Green A, et al. Global prevalence of diabetes: estimates for the year 2000 and projections for 2030. Diabetes Care 2004:27:1047-53.

3. Wright RS, Anderson JL, Adams CD, et al. 2011 ACCF/AHA Focused Update of the Guidelines for the Management of Patients With Unstable Angina/Non-ST-Elevation Myocardial Infarction (Updating the 2007 Guideline): a report of the American College of Cardiology Foundation/American Heart Association Task Force on Practice Guidelines. Circulation 2011;123:2022-60.

4. Kushner FG, Hand M, Smith SC Jr, et al. 2009 Focused Updates: ACC/AHA Guidelines for the Management of Patients With ST-Elevation Myocardial Infarction (updating the 2004 Guideline and 2007 Focused Update) and ACC/AHA/SCAI Guidelines on Percutaneous Coronary Intervention (updating the 2005 Guideline and 2007 Focused Update): a report of the American College of Cardiology Foundation/American Heart Association Task Force on Practice Guidelines. Circulation 2009;120:2271-306.

5. Hamm CW, Bassand JP, Agewall S, et al. ESC Guidelines for the management of acute coronary syndromes in patients presenting without persistent ST-segment elevation: The Task Force for the management of acute coronary syndromes (ACS) in patients presenting without persistent ST-segment elevation of the European Society of Cardiology (ESC). Eur Heart J 2011;32:2999-3054.

6. Van de Werf F, Bax J, Betriu A, et al. Management of acute myocardial infarction in patients presenting with persistent ST-segment elevation: the Task Force on the Management of ST-Segment Elevation Acute Myocardial Infarction of the European Society of Cardiology. Eur Heart J 2008;29:2909-45.

7. Hasdai D, Behar S, Wallentin L, et al. A prospective survey of the characteristics, treatments and outcomes of patients with acute coronary syndromes in Europe and the Mediterranean basin; the Euro Heart Survey of Acute Coronary Syndromes (Euro Heart Survey ACS). Eur Heart J 2002;23:1190-201.

8. Budaj A, Brieger D, Steg PG, et al. Global patterns of use of antithrombotic and antiplatelet therapies in patients with acute coronary syndromes: insights from the Global Registry of Acute Coronary Events (GRACE). Am Heart J 2003;146:999-1006.

9. Fox KA, Goodman SG, Klein W, et al. Management of acute coronary syndromes. Variations in practice and outcome; findings from the Global Registry of Acute Coronary Events (GRACE). Eur Heart J 2002;23:1177-89.

10. Gibson CM. NRMI and current treatment patterns for ST-elevation myocardial infarction. Am Heart J 2004;148(5 Suppl):S29-33.

11. Steg PG, Goldberg RJ, Gore JM, et al. Baseline characteristics, management practices, and in-hospital outcomes of patients hospitalized with acute coronary syndromes in the Global Registry of Acute Coronary Events (GRACE). Am J Cardiol 2002;90:358-63. 
12. Yusuf S, Flather M, Pogue J, et al. Variations between countries in invasive cardiac procedures and outcomes in patients with suspected unstable angina or myocardial infarction without initial ST elevation. OASIS (Organisation to Assess Strategies for Ischaemic Syndromes) Registry Investigators. Lancet 1998;352:507-14.

13. Fox KA, Goodman SG, Anderson FA Jr, et al. From guidelines to clinical practice: the impact of hospital and geographical characteristics on temporal trends in the management of acute coronary syndromes. The Global Registry of Acute Coronary Events (GRACE). Eur Heart J 2003;24:1414-24.

14. Polonski L, Gasior M, Gierlotka M, et al. Polish Registry of Acute Coronary Syndromes (PL-ACS). Characteristics, treatments and outcomes of patients with acute coronary syndromes in Poland. Kardiol Pol 2007;65:861-72; discussion 73-4.

15. Chew DP, Amerena J, Coverdale S, et al. Current management of acute coronary syndromes in Australia: observations from the acute coronary syndromes prospective audit. Intern Med J 2007;37:741-8.

16. Goodman SG, Huang W, Yan AT, et al. The expanded Global Registry of Acute Coronary Events: baseline characteristics, management practices, and hospital outcomes of patients with acute coronary syndromes. Am Heart J 2009;158:193-201.e1-5.

17. Daida $\mathrm{H}$, Miyauchi $\mathrm{K}, \mathrm{Ogawa} \mathrm{H}$, et al. Management and two-year long-term clinical outcome of acute coronary syndrome in Japan: prevention of atherothrombotic incidents following ischemic coronary attack (PACIFIC) registry. Circ J 2013;77:934-43.

18. Xavier D, Pais P, Devereaux PJ, et al. Treatment and outcomes of acute coronary syndromes in India (CREATE): a prospective analysis of registry data. Lancet 2008;371:1435-42.

19. ACCESS Investigators. Management of acute coronary syndromes in developing countries: acute coronary events-a multinational survey of current management strategies. Am Heart $J$ 2011;162:852-9.e22.

20. Alhabib KF, Hersi A, Alfaleh $\mathrm{H}$, et al. Baseline characteristics, management practices, and in-hospital outcomes of patients with acute coronary syndromes: results of the Saudi project for assessment of coronary events (SPACE) registry. J Saudi Heart Assoc 2011;23:233-9.

21. Gibson CM, Pride YB, Frederick PD, et al. Trends in reperfusion strategies, door-to-needle and door-to-balloon times, and in-hospital mortality among patients with ST-segment elevation myocardial infarction enrolled in the National Registry of Myocardial Infarction from 1990 to 2006. Am Heart J 2008;156:1035-44.

22. Santos JF, Aguiar C, Gavina C, et al. Portuguese Registry of Acute Coronary Syndromes: seven years of activity. Rev Port Cardiol 2009;28:1465-500.

23. Eagle KA, Nallamothu $\mathrm{BK}$, Mehta $\mathrm{RH}$, et al. Trends in acute reperfusion therapy for ST-segment elevation myocardial infarction from 1999 to 2006: we are getting better but we have got a long way to go. Eur Heart J 2008;29:609-17.

24. Alhabib KF, Sulaiman K, Al-Motarreb A, et al. Baseline characteristics, management practices, and long-term outcomes of Middle Eastern patients in the Second Gulf Registry of Acute Coronary Events (Gulf RACE-2). Ann Saudi Med 2012;32:9-18.

25. Karrowni W, Abdallah $\mathrm{M}$, Itani $\mathrm{S}$, et al. Management of acute coronary syndromes in developing countries: are we complying with practice guidelines? Int J Cardiol 2010;144:95-6.

26. Peterson ED, Shah BR, Parsons L, et al. Trends in quality of care for patients with acute myocardial infarction in the National Registry of Myocardial Infarction from 1990 to 2006. Am Heart $J$ 2008;156:1045-55

27. Ahmad WA, Ramesh SV, Zambahari R. Malaysia-ACute CORonary syndromes Descriptive study (ACCORD): evaluation of compliance with existing guidelines in patients with acute coronary syndrome. Singapore Med J 2011;52:508-11.

28. Cheng $\mathrm{Cl}$, Chen $\mathrm{CP}$, Kuan PL, et al. The causes and outcomes of inadequate implementation of existing guidelines for antiplatelet treatment in patients with acute coronary syndrome: the experience from Taiwan Acute Coronary Syndrome Descriptive Registry (T-ACCORD Registry). Clin Cardiol 2010;33:E40-8.

29. Canto AJ, Kiefe $\mathrm{Cl}$, Goldberg RJ, et al. Differences in symptom presentation and hospital mortality according to type of acute myocardial infarction. Am Heart J 2012;163:572-9.

30. Chong $\mathrm{E}$, Shen L, Tan HC, et al. A cohort study of risk factors and clinical outcome predictors for patients presenting with unstable angina and non ST segment elevation myorardial infraction undergoing coronary intervention. Med J Malaysia 2011;66:249-52.

31. Park HW, Yoon $\mathrm{CH}$, Kang SH, et al. Early- and late-term clinical outcome and their predictors in patients with ST-segment elevation myocardial infarction and non-ST-segment elevation myocardial infarction. Int J Cardiol 2013;169:254-61. 


\section{Correction}

Kassaian SE, Masoudkabir F, Sezavar H, et al. Clinical characteristics, management and 1-year outcomes of patients with acute coronary syndrome in Iran: the Iranian Project for Assessment of Coronary Events 2 (IPACE2). BMJ Open 2015;5:e007786.

The institutional affiliation of $\mathrm{Dr}$ Ali Pourmoghaddas should be: Isfahan Cardiovascular Research Center, Cardiovascular Research Institute, Isfahan University of Medical Sciences, Isfahan, Iran. In addition, the first name of the co-author Bahin Pourmirza is misspelt and should be 'Behin'.

BMJ Open 2016;6:e007786corr1. doi:10.1136/bmjopen-2015-007786corr1

CrossMark 


\section{Correction}

Kassaian SE, Masoudkabir F, Sezavar H, et al. Clinical characteristics, management and 1-year outcomes of patients with acute coronary syndrome in Iran: the Iranian Project for Assessment of Coronary Events 2 (IPACE2). BMJ Open 2015;5:e007786. There is a misspelling of the sixth author's name in this paper. The author's correct name is Javad Kojuri.

BMJ Open 2016;6:007786corr2. doi:10.1136/bmjopen-2015-007786corr2

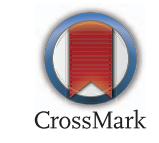

